\title{
Antitumoral effect of Ocoxin in hepatocellular carcinoma
}

\author{
ELENA DÍAZ-RODRÍGUEZ ${ }^{1}$, AL-MAHY EL-MALLAH ${ }^{1,3}$, EDUARDO SANZ $^{2}$ and ATANASIO PANDIELLA ${ }^{1}$ \\ ${ }^{1}$ Institute of Molecular and Cellular Cancer Biology, \\ CSIC-University of Salamanca, 37007 Salamanca; ${ }^{2}$ Catalysis, S.L., 28016 Madrid, Spain
}

Received July 13, 2016; Accepted November 23, 2016

DOI: $10.3892 / 01.2017 .6440$

\begin{abstract}
Hepatocellular carcinoma (HCC) is becoming one of the most prevalent types of cancer worldwide. The most efficient types of treatment at present include surgical resection and liver transplantation, but these treatments may only be used in a small percentage of patients. In order to identify novel therapeutic strategies for this disease, the present study explored the potential antitumoral effect of Ocoxin ${ }^{\circledR}$ oral solution (OOS) in HCC. OOS inhibited the proliferation of HCC cell lines in a time- and dose-dependent manner, being more efficient when used in combination with sorafenib, a standard of care treatment for patients diagnosed with advanced-stage disease. Mechanistic studies indicated that the effect of OOS was due to the induction of cell cycle arrest rather than the stimulation of apoptotic cell death. The cell cycle was slowed down in all phases in the HCC cell lines treated with OOS. Finally, when tested in animal models of HCC, OOS reduced tumor progression through the induction of necrosis in xenograft tumor models. Considering the poor prognosis and high resistance to antitumor treatments of $\mathrm{HCC}$, the antiproliferative action of OOS, particularly in combination with sorafenib, provides the opportunity to investigate the effect of combined therapy in a clinical setting.
\end{abstract}

\section{Introduction}

Hepatocellular carcinoma (HCC) is the most common type of liver cancer and is rapidly becoming one of the most prevalent types of cancer, being the sixth most common type of neoplasm worldwide and the third leading cause of cancer-associated mortality, responsible for more than 650,000 mortalities per year globally (1). The most efficient treatments for this disease

Correspondence to: Dr Elena Díaz-Rodríguez or Dr Atanasio Pandiella, Institute of Molecular and Cellular Cancer Biology-CIC, Campus Miguel de Unamuno s/n, 37007 Salamanca, Spain

E-mail: ediaz@usal.es

E-mail: atanasio@usal.es

Current address: ${ }^{3}$ Zoology Department, Faculty of Science, Beni-Suef University, Beni Suef 62511, Egypt

Key words: hepatocellular carcinoma, antioxidants, cell cycle include complete surgical resection of the tumor and liver transplantation. The outcome of HCC is typically poor since only $10-20 \%$ of HCCs may be completely removed by surgery and the lack of complete removal leads to the relapse of the disease (2).

$\mathrm{HCC}$ is a tumor characterized by being highly resistant to systemic chemotherapy. The use of the kinase inhibitor sorafenib has previously increased the overall survival of patients suffering from this disease (3-6). This agent, which may be orally administered, has antiproliferative and antiangiogenic activity and delays tumor progression, being at present the standard of care in patients diagnosed with an advanced-stage disease (7). However, the active search for other treatments that may be used to treat this malignancy is underway. Thus, during previous years, several phase III trials, including patients with intermediate and advanced-stages, have been carried out to test the effects of various drugs; however, in general, they have failed to present survival benefits (5), driving the search for the identification of novel oncogenic drivers and molecular therapeutics that may be used to treat HCC. In the previous decade, molecular analyses of patients with HCC have resulted in the identification of several genomic subclasses (5), although, thus far, there is no clear consensus with respect to the association between molecular subclass and patient outcome.

Several precedents indicate that natural products may be useful anticancer supplements. Numerous studies have indicated that green tea-derived polyphenol epigallocatechin-3-gallate exerts chemopreventive and hepatoprotective effects against HCC in preclinical models (8-10). The mechanism of action of this product has not been fully elucidated, but may include the reduction of prostaglandin biosynthesis by HCC cells (11), which may then induce apoptotic cell death in HCC. In addition, epigallocatechin-3-gallate may reduce the metastatic ability of HCC cells by decreasing the production of osteopontin (12).

Ocoxin ${ }^{\circledR}$ oral solution (OOS) is an oral nutritional supplement that contains several compounds with anticancer activities, including epigallocatechin-3 gallate (13). In addition, OOS is comprised of a variety of other components, including vitamins $\mathrm{B} 6$ and $\mathrm{C}$ and cinnamic acid, which exhibit anticancer properties (14-16). In addition, OOS contains glycyrrhizinic acid, which exhibits anti-inflammatory and immunomodulatory effects (17). As OOS contains several compounds with an antitumor action, it is currently being investigated in clinical trials as part of the treatment of several types of cancer, in 
which an improvement in the quality of life of the patients has been reported $(18,19)$. Additionally, several recent reports have demonstrated that OOS exhibits in vitro and in vivo antitumoral action in various tumor models, including HER2 positive breast cancer (13) and acute myeloid leukemia (20). These two tumor types are dissimilar at the molecular and cellular levels; however, the clear antitumoral action of OOS has been reported in the two. In addition, the antitumor action appeared to have been mediated by the slowing of cell cycle progression induced by an increase in expression of the cell cycle inhibitor p27. Furthermore, a recent clinical study revealed preliminary data that suggested that OOS may be useful in patients with end-stage HCC (18).

On the basis of these precedents, the present study aimed to explore the potential antitumoral effect of OOS in preclinical models of HCC. The present study demonstrated that OOS exhibits antitumoral effects in various HCC cellular models in vitro. In addition, when tested in vivo, an antitumoral effect was also demonstrated in terms of tumor growth reduction. Combinational studies with sorafenib, the standard of care for the treatment of $\mathrm{HCC}$, indicated increased antitumoral activity in the combination, providing the rationale to additionally explore the clinical value of this therapy in HCC.

\section{Materials and methods}

Reagents and antibodies. The Dulbecco's modified Eagle's medium (DMEM), MTT, hematoxylin and eosin were purchased from Sigma-Aldrich (Merck Millipore, Darmstadt, Germany). The fetal bovine serum (FBS) and antibiotics were purchased from Thermo Fisher Scientific, Inc. (Waltham, MA, USA) and Immobilon P polyvinylidene fluoride membranes from Merck Millipore. OOS was provided by Catalysis, S.L. (Madrid, Spain). The other generic chemicals were purchased from Sigma-Aldrich, Roche Applied Science (Mannheim, Germany) or Merck Millipore.

The origins of the various antibodies used in the Western blot analyses are as follows: Anti-GAPDH (cat. no. sc-166574; 1:10,000) and anti-cyclin B (cat. no. sc-245; 1:5,000) antibodies were purchased from Santa Cruz Biotechnology, Inc. (Dallas, TX, USA) and the anti-phospho-histone H3 (cat. no. 06-570; 1:3,500) from Merck Millipore. The horseradish peroxidase-conjugated secondary antibodies (goat anti-rabbit; cat. no. 170-6515; 1:20,000; and goat anti-mouse; cat. no. 170-6516; 1:10,000), were obtained from Bio-Rad Laboratories Inc. (Hercules, CA, USA).

Cell culture, cell proliferation measurement, protein purification and western blot analysis. The HepG2 and Huh7 HCC cell lines were donated by Dr Mollinedo (University of Salamanca, Salamanca, Spain) and grown in DMEM supplemented with $10 \% \mathrm{FBS}$ and antibiotics. The cells were cultured at $37^{\circ} \mathrm{C}$ in a humidified atmosphere in the presence of $5 \% \mathrm{CO}_{2}-95 \%$ air.

The proliferation of HCC cells was examined using a modified MTT metabolization assay (13). The cells were plated in triplicate and treated the next day as indicated, with various concentrations of OOS alone or in combination with other drugs: Sorafenib (LC Laboratories, Woburn, MA, USA) $0.1,0.3,1$ or $3 \mu \mathrm{M}$; docetaxel (Hospira UK Ltd., Leamington Spa, UK) 1 or $3 \mu \mathrm{M}$; and cisplatin (Pharmacia Grupo Pfizer,
Madrid, Spain) 2 and $20 \mu \mathrm{M}$. On the day of the experiment, MTT was added to the wells at $0.5 \mathrm{mg} / \mathrm{ml}$ and incubated at $37^{\circ} \mathrm{C}$ for $1 \mathrm{~h}$. The MTT-formazan crystals were dissolved in DMSO and the absorbance of the samples was recorded at $570 \mathrm{~nm}$ using a Tecan spectrophotometer with X-Fluor 4 (version 4.50; Tecan Trading AG, Männedorf, Switzerland) software. A minimum of 3 wells were analyzed for each condition, and the results are presented as the mean \pm standard deviation (SD) of a representative experiment repeated at least twice. In order to prepare the cells for protein analyses, they were washed in PBS and lysed in an ice-cold lysis buffer $(140 \mathrm{mM} \mathrm{NaCl}, 10 \mathrm{mM}$ EDTA, 10\% glycerol, 11\% Nonidet P-40, 20 mM Tris, $\mathrm{pH} 8.0$, $1 \mathrm{mM}$ pepstatin, $1 \mu \mathrm{g} / \mathrm{ml}$ aprotinin, $1 \mu \mathrm{g} / \mathrm{ml}$ leupeptin, $1 \mathrm{mM}$ PMSF and $1 \mathrm{mM}$ sodium orthovanadate). Protein quantification, SDS-PAGE and western blot analysis were performed as previously described (21). For the western blot analysis, a total of $50 \mu \mathrm{g}$ protein were probed with the indicated antibodies.

Transfection and fluorimetric quantification. The HepG2 and Huh7 cells were transfected with a plasmid coding for the luciferase gene (pCDNA3.1-Luc) using the jetPEI ${ }^{\circledR}$ reagent (Polyplus-transfection ${ }^{\circledR}$, Illkirch, France) according to the protocol of the manufacturer. The plasmid coding for the luciferase gene (pCDNA3.1-Luc) was donated by Dr J. Massagué (Memorial Sloan-Kettering Cancer Center, New York, NY, USA). Positive transfected cells were obtained subsequent to selection with the appropriate antibiotic, $450 \mu \mathrm{g} / \mathrm{ml}$ hygromycin . Once the clones had been isolated they were independently harvested by cloning rings, grown in multiwell plates and expanded. Hygromycin positive clones were tested for the presence of the luciferase gene by adding $150 \mu \mathrm{g} / \mathrm{ml}$ luciferin to the culture media, and then reading the luminescence in triplicate wells using a Synergy4 multi-mode microplate reader with Gen5 1.05 software (BioTek Instruments, Inc., Winooski, VT, USA). Each clone was analyzed at least twice and one of them, HepG2-Luc\#22, was chosen for further experiments. In addition, a previously published MCF7-lucifesase expressing clone was established as previously described (22) and analyzed in parallel as a positive control. The correct behavior of these clones in terms of general aspect, proliferation (measured as MTT metabolization) and cell cycle profile was determined before further proceeding.

Cell death, cell cycle and cell synchronization and release experiments. To analyze apoptotic cell death, the cells were treated for $48 \mathrm{~h}$ with the indicated treatments (OOS; dilutions, $1: 25,1: 50$ or 1:100 in culture media), resuspended in binding buffer (10 mM 4-(2-hydroxyethyl)-1-piperazineethanesulphonic acid/NaOH; $140 \mathrm{mM} \mathrm{NaCl} ; 2,5 \mathrm{nM} \mathrm{CaCl}_{2} ; \mathrm{pH}=7.4$ ) containing $5 \mu \mathrm{l}$ Annexin $\mathrm{V}$-fluorescein isothiocyanate (BD Biosciences) and $5 \mu \mathrm{l} 50 \mu \mathrm{g} / \mathrm{ml}$ propidium iodide (PI), and stained at room temperature for $15 \mathrm{~min}$. A total of 50,000 cells were acquired using a BD Accuri C6 flow cytometer and C6 software (version 1.0.264.21; BD Biosciences).

For cell cycle analysis by flow cytometry, the ethanol-fixed cells were stained with $5 \mu \mathrm{g} / \mathrm{ml}$ PI and $250 \mu \mathrm{g}$ DNase-free RNase. A total of 50,000 cells were acquired as previously described. For the cell cycle synchronization experiments, the cells were arrested at various phases of the cell cycle as previously described (21). Thus, for $\mathrm{G}_{2}-\mathrm{M}$ synchronization, 
nocodazole treatment was used, whereas for $\mathrm{G}_{1}-\mathrm{S}$ arrest a double thymidine block was carried out. Subsequent to synchronization, the cells were washed twice in PBS and released in normal medium, or in medium supplemented with OOS (dilution, 1:25), and the cell samples were harvested after $1,2,3,6,9,12$ or $24 \mathrm{~h}$ for additional flow cytometry or western blot analysis.

In vivo experiments. For the animal studies, 12 7-week-old female athymic mice (BALB/C nu/nu) weighing between 18 and $20 \mathrm{~g}$, were purchased from Charles River Laboratories (Wilmington, MA, USA) and kept in pathogen-free housing at Universidad de Salamanca Animal Care Facility (Salamanca, Spain). All animal experiments were performed according to the institutional guidelines and protocol approved by the Ethics Committee of Universidad de Salamanca (Salamanca, Spain). A total of 1 week subsequent to the purchase of the mice, they were injected with the appropriate cells to generate tumors. For the xenograft model, $1 \times 10^{6} \mathrm{HepG} 2$ cells resuspended in $50 \mu 1 \mathrm{DMEM}$ and $50 \mu \mathrm{l}$ Matrigel were subcutaneously injected into the right caudal flank of each mouse. When the tumors became palpable, the mice were randomly assigned into two groups (six per group), which received either vehicle alone, the control group, or $100 \mu \mathrm{l}$ OOS per $20 \mathrm{~g}$ weight of mouse. The treatments were administered with a once daily schedule (Monday to Friday) by oral gavage until the humane endpoint (tumor volume, $2.000 \mathrm{~mm}^{3}$ ) was achieved by the control group and they were subsequently sacrificed by isofluorane euthanasia. The mice were weighed and the tumors were measured twice a week with a digital caliper (Proinsa, Vitoria, Spain). Tumor volumes were calculated using the formula: $\mathrm{V}=(\mathrm{L} / 2)$ $\mathrm{x}(\mathrm{W} / 2)^{2} \times 4 / 3 \times \pi$, where $\mathrm{V}=$ volume (cubic $\mathrm{mm}$ ), $\mathrm{L}=$ length $(\mathrm{mm})$ and $\mathrm{W}=$ width $(\mathrm{mm})$. Subsequent to the sacrifice of the mice, the tumor tissue was resected and immediately frozen at $-80^{\circ} \mathrm{C}$.

For the disseminated model, HepG2-Luc cells were directly injected into the liver of $14 \mathrm{BALB} / \mathrm{C}$ nu/nu mice who were anesthetized with ketamine and xylazine, and a 5-mm incision was carried out in the upper left ventral region of the abdomen, through the skin and the muscle layer to access the abdominal cavity. A total of $0.5 \times 10^{6} \mathrm{HepG} 2$-Luc cells resuspended in $25 \mu \mathrm{l}$ DMEM and $25 \mu \mathrm{l}$ Matrigel were injected in the liver, which was observed through the incision. The muscle and the skin layers were independently sutured, the region was cleaned and the mice were treated with buprenorfine, used as an analgesic. The correct localization of the cells was analyzed in vivo by bioluminescent imaging, performed with the IVIS 50 imaging system (Xenogen Corporation, Alameda, CA, USA), and the results were analyzed using Living Image software (version 4.1; Perking Elmer, Boston, MA, USA). The mice were intraperitoneally injected with $150 \mathrm{mg} / \mathrm{kg}$ aqueous solution of D-luciferin (Perking Elmer, Boston, MA, USA) and anesthetized by isoflurane inhalation, and images were captured. The status of the mice, including general status and weight, and tumor growth were determined twice a week.

Histological and immunohistochemical (IHC) analyses. Representative tumor areas were fixed in formalin, embedded in paraffin, cut into $2-3-\mu \mathrm{m}$ sections and either stained with hematoxylin and eosin or prepared for IHC, which was performed as previously described (23). Thus, two cell conditioning periods of $8 \mathrm{~min}$ at $95^{\circ} \mathrm{C}$ and $4 \mathrm{~min}$ at $100^{\circ} \mathrm{C}$ on a hot plate using Tris-EDTA, $\mathrm{pH}=8$, buffer were performed on previously dewaxed formalin-fixed paraffin-embedded tissue sections. Sections were incubated for $42 \mathrm{~min}$ at $37^{\circ} \mathrm{C}$ with a 1:50 dilution anti-Ki67 antibody (Master Diagnóstica, Granada, Spain; cat. no. MAD-000310QD), and the staining was performed with the IHC 3,3'-diaminobenzidine system (Ventana Medical Systems, Tuscon, AZ, USA). The results were evaluated by pathologists blinded to the clinicopathological and molecular data. The extension of the necrotic area present in each tissue sample was assessed by measuring the total tumor and necrotic areas. The tumors were scanned and the percentage of the necrotic region was normalized to the total area using the dotSlide system and dotSlide 2.1 software (Olympus Corporation, Tokyo, Japan). These procedures were performed by independent personnel of the pathology unit of the University of Salamanca (Salamanca, Spain).

Statistical analysis. Each condition was analyzed in triplicate or quadruplicate and data are presented as the mean \pm SD of $\geq 3$ independent experiments. Comparisons of continuous variables between two groups were performed using a two-sided Student's $t$ test. $\mathrm{P}<0.05$ was considered to indicate a statistically significant difference.

\section{Results}

Effect of OOS on the proliferation of HCC cell lines. To study the potential effect of OOS on HCC, HepG2 and Huh7 HCC cell lines were treated for various days with increasing amounts of OOS, and the cellular response in terms of cellular proliferation was assessed. In those conditions, OOS reduced MTT metabolization, used as an indicator of cell proliferation, in the two cell lines (Fig. 1). The effect was time- and dose-dependent. The HepG2 cell line was more sensitive to the antiproliferative effect of OOS, compared with the Huh7 cell line. Treatment of HepG2 cells with a 1:100 dilution of OOS inhibited cell proliferation by $50 \%$ subsequent to three days of treatment $(\mathrm{P}=0.00082$; Fig. $1 \mathrm{~A})$, whereas an equal dose of OOS did not substantially affect the proliferation of Huh7 cells $(\mathrm{P}=0.21$; Fig. 1B). Due to the higher sensitivity of the HepG2 cell line to OOS, the present study preferentially used the aforementioned cell line as the principal model to explore the mechanism of action of OOS in HCC.

Effect of OOS in combination with other anti-HCC treatments. In the majority of the cases the success of antitumoral treatments is based on the combination of various agents. For that reason, the present study investigated if the antitumoral effect of OOS on the HepG2 cell line was increased by other drugs commonly used in the treatment of cancer. Since sorafenib is the recommended systemic treatment for patients diagnosed with advanced HCC, the present study tested the effect of OOS in combination with sorafenib. The simultaneous use of the agents was more efficient with respect to inhibiting the cell growth, compared with any of the individual treatments (Fig. 2A). OOS was also evaluated in combination with other drugs that had exhibited efficacy in the treatment of patients with HCC, including the antitumoral agents cisplatin (24) and 

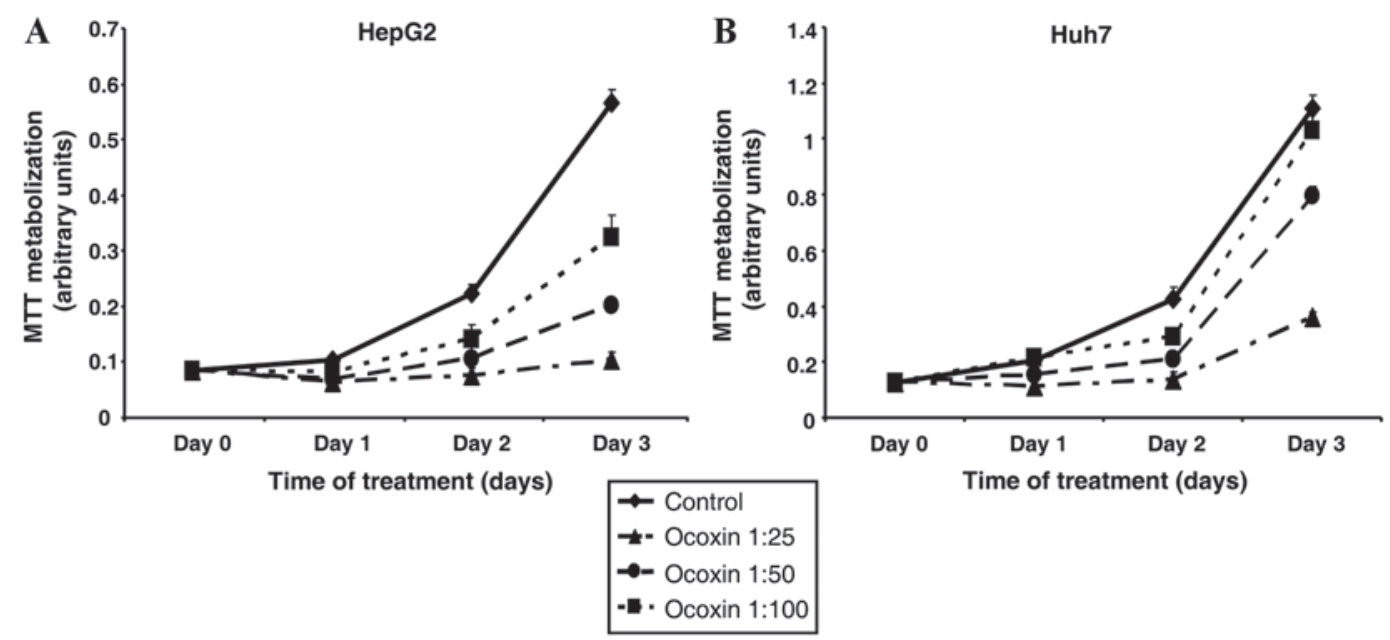

Time of treatment (days)

Figure 1. OOS treatment impairs proliferation of HCC cell lines. (A) HepG2 or (B) Huh7 cells were incubated for $\leq 3$ days with increasing concentrations of OOS diluted in medium, and cell proliferation was measured in terms of MTT metabolization. For each cell line, the data are presented as the mean \pm standard deviation of triplicates of a representative experiment that was repeated $\geq 3$ times. OOS, Ocoxin ${ }^{\circledR}$ oral solution.

docetaxel $(25,26)$. However, treatment with OOS in combination with these agents did not substantially improve the effect of the individual treatments (Fig. 2B).

Effect of OSS on apoptotic cell death and cell cycle progression. A decrease in MTT metabolization may be caused by an increase in cell death, cell cycle arrest, or a combination of the two. To additionally investigate the mechanism of action of OOS in HCC cells, apoptotic cell death was initially assessed. The HepG2 cells were treated with increasing concentrations of OOS and apoptotic cell death was determined by double staining with Annexin V and PI. No major changes in the various populations were observed in any of the concentrations tested, indicating that there was no apoptotic cell death induced by OOS observed under the experimental conditions of the present study (Fig. 3A).

The current study then investigated if OOS induced cell cycle arrest in HCC cells. The cells treated for $48 \mathrm{~h}$ with increasing concentrations of OOS were fixed and the cell cycle profile was analyzed by PI staining of the DNA. A decrease in the number of cells in the $G_{1}$ phase of the cell cycle was observed. In addition, treatment with OOS resulted in an increase in the number of cells present in the $\mathrm{G}_{2}-\mathrm{M}$ section of the histogram (Fig. 3B). These results indicated that the antitumoral action of OOS was due to an effect on cell cycle progression, without an apparent effect of OOS on cell viability.

To more precisely map the effect of OOS on cell cycle progression, synchronization and release experiments were carried out. Two types of synchronization procedures were used to arrest cells in various cell cycle phases. Firstly, HepG2 cells were synchronized in the $\mathrm{G}_{1}$ phase of the cell cycle by double thymidine treatment (21), and then released from the double thymidine block by incubation in normal medium, or in medium supplemented with OOS. A section of the sample was fixed to analyze cell cycle progression by flow cytometry, and the other section was lysed in order to explore the biochemical markers of each cell cycle phase using western blotting. Cells were observed to progress through the cell cycle more slowly subsequent to OOS treatment (Fig. 4A and B). For example, when the cell cycle distribution was analyzed using flow cytometry $9 \mathrm{~h}$ subsequent to releasing the block, the majority of the cells had completed the cell cycle in the control condition, as the majority of the cells were already in the $G_{1}$ phase of the cell cycle, with $2 \mathrm{~N}$ DNA content. At that time, the majority of the OOS-treated cells were in the $\mathrm{G}_{2}-\mathrm{M}$ phase, with 4N DNA content (Fig. 4A). This observation was confirmed when the expression levels of key cell cycle markers were determined by western blot analysis. Thus, at the aforementioned time point, the levels of the $\mathrm{G}_{2}-\mathrm{M}$ markers cyclin $\mathrm{B}$ and phospho-histone $\mathrm{H} 3$ were higher in the OOS-treated cells, compared with the control population, and demonstrated a larger number of cells in the $\mathrm{G}_{2}-\mathrm{M}$ phase (Fig. 4B).

The delay of the cell cycle progression induced by OOS was additionally confirmed by nocodazole synchronization experiments. This is a reversible microtubule polymerization inhibitor that arrests the cell cycle in the $\mathrm{M}$ phase, since cells are unable to form the mitotic spindle in the presence of nocodazole and the spindle checkpoint becomes active (27). When HepG2 cells were synchronized using nocodazole and released into OOS-containing media, a delay in mitotic exit was observed by flow cytometry and by western blot analysis of key mitotic markers (Fig. 4C and D). A total of $1 \mathrm{~h}$ subsequent to the release into the media, $1 / 2$ of the cells were in $G_{1}$ in the control condition, whereas none of the cells had reached $\mathrm{G}_{1}$ in the OOS-treated group as observed by flow cytometry cell cycle analysis (Fig. 4C). In addition, a delay in the degradation of the $\mathrm{G}_{2}-\mathrm{M}$ markers cyclin $\mathrm{B} 1$ and phospho-histone $\mathrm{H} 3$ by western blot analysis was observed subsequent to OOS treatment, confirming that the cell cycle was slowed under that condition (Fig. 4D).

Effect of OOS treatment on xenograft tumor models. To determine if OOS exerted an effect on $\mathrm{HCC}$ growth in vivo, two distinct mouse models were developed. In the first one, HepG2 cells were engrafted in the flanks of BALB/c nu/nu athymic mice. Subsequent to three days, when the tumors became palpable, the mice were randomly assigned into two groups, 

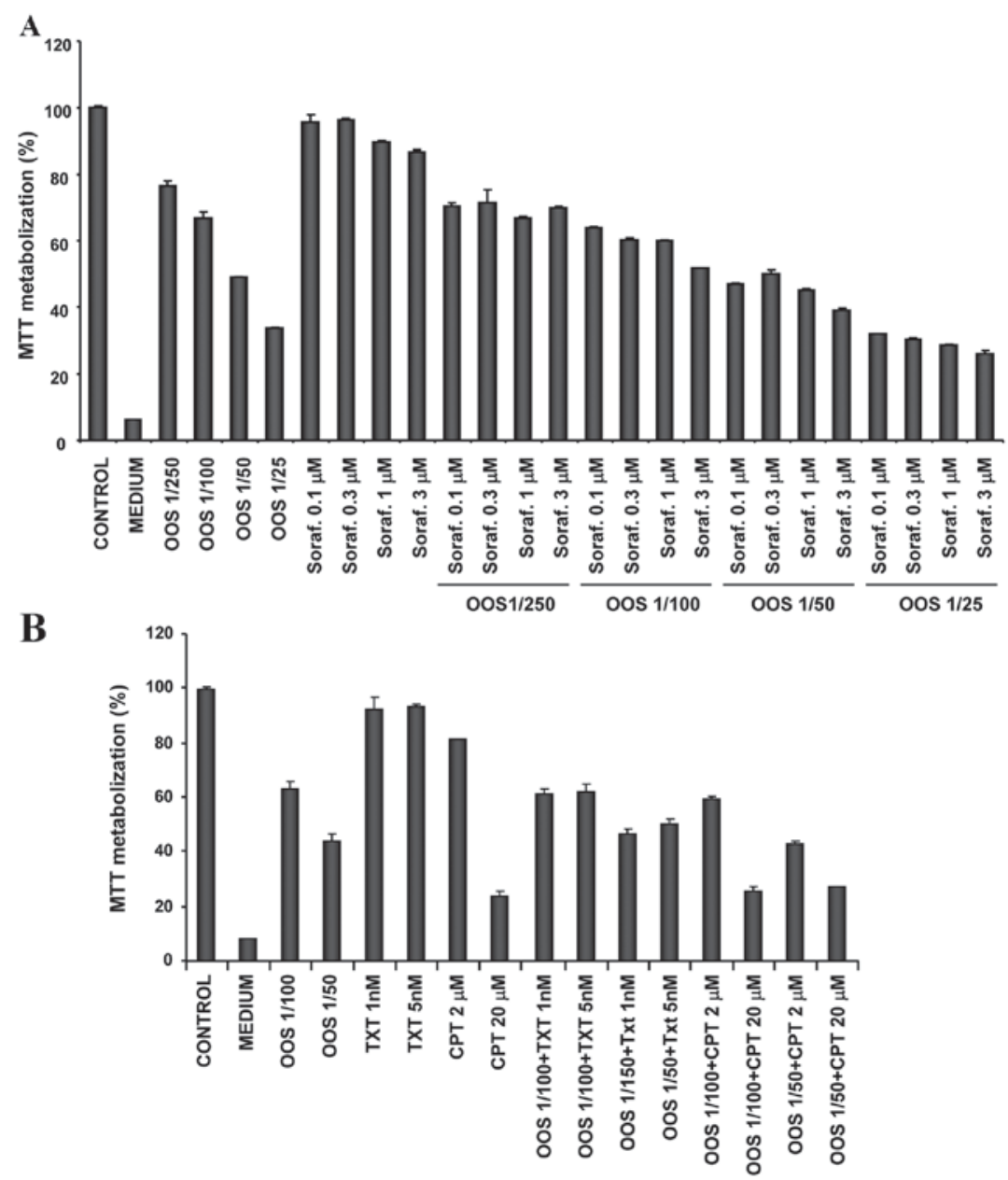

Figure 2. Effect of OOS in combination with other drugs in hepatocellular carcinoma. (A) OOS is more effective when used in combination with sorafenib. HepG2 cells were treated for $48 \mathrm{~h}$ with increasing concentrations of OOS, sorafenib or their combination, and the effect was determined by MTT uptake. (B) Similarly, the effect of OOS in combination with the antitumoral drugs CPT or TXT was determined. Mean absorbance values of untreated samples were taken as $100 \%$ and the mean values were then referred to that. Data are presented as the mean \pm standard deviation of triplicates of an experiment that was repeated $\geq 3$ times. CPT, cisplatin; TXT, docetaxel; OOS, Ocoxin ${ }^{\circledast}$ oral solution; soraf, sorafenib.
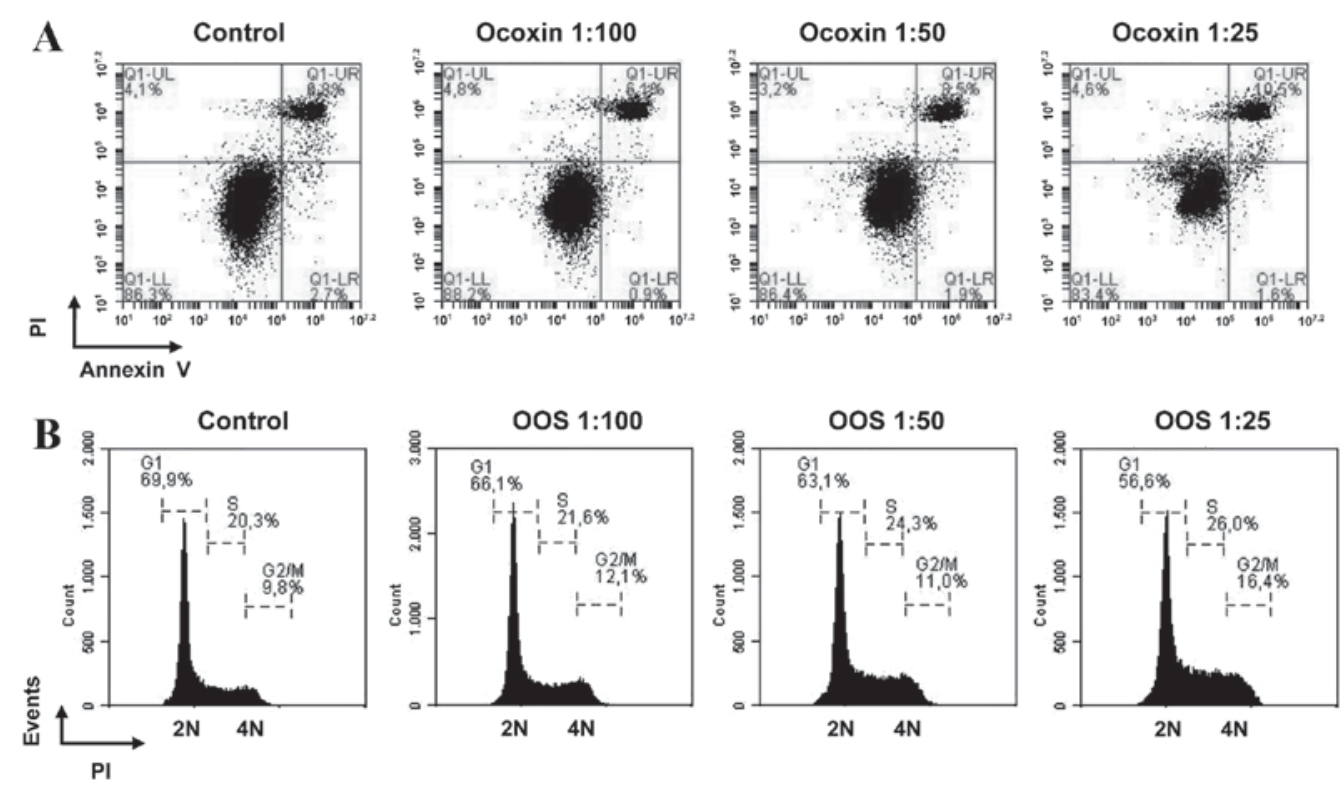

Figure 3. OOS does not induce apoptotic cell death or cell cycle arrest in vitro. (A) HepG2 cells were treated with the indicated concentrations of OOS and, subsequent to $48 \mathrm{~h}$, were harvested and stained with Annexin V and PI to determine apoptotic cell death. (B) OOS does not induce mayor cell cycle arrest. Asynchronously growing HepG2 cells were treated for $48 \mathrm{~h}$ with the indicated dilution of OOS. Cells were then harvested, ethanol-fixed and their cell cycle status determined by PI staining. OOS, Ocoxin ${ }^{\circledR}$ oral solution; PI, propidium iodide. 

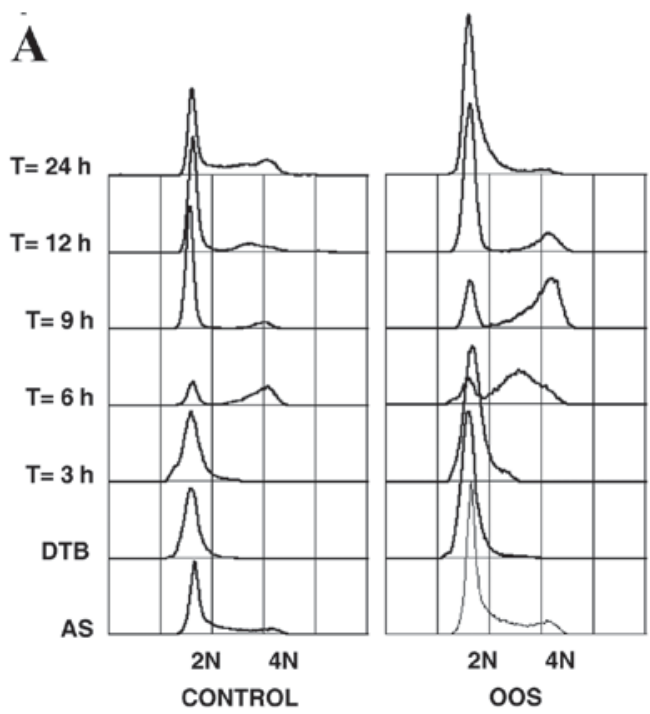

B
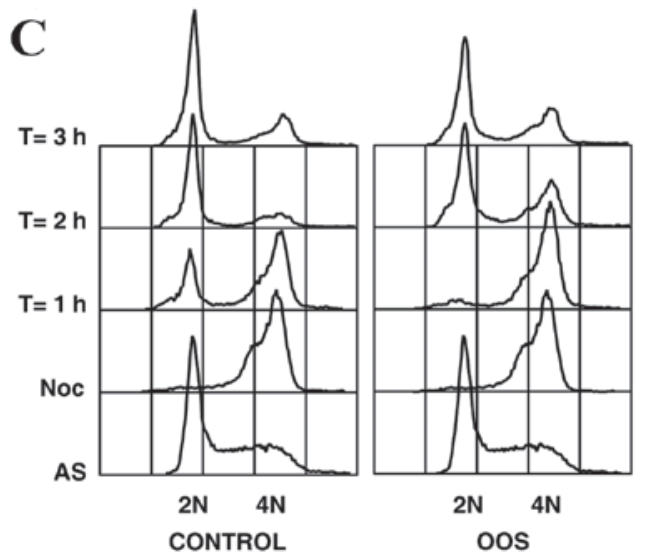

D

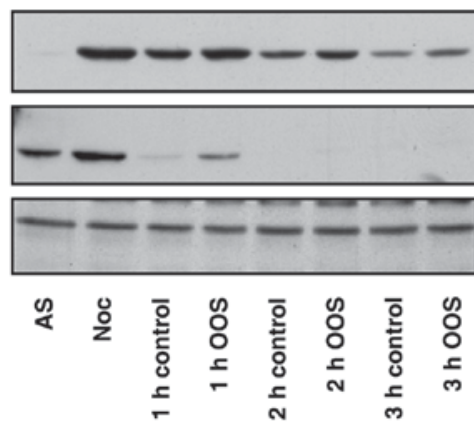

pHistone $\mathrm{H3}$

Cyclin B1

GAPDH

Figure 4. OOS induces cell cycle retardation. OOS induces cell cycle arrest. HepG2 cells were arrested in the $\mathrm{G}_{1}-\mathrm{S}$ phase by a double thymidine block, released into normal or OOS-supplemented medium as aforementioned and collected at the indicated time points. A section of the sample was fixed for DNA content determination by flow cytometry (A) and the other section was lysed for protein preparation (B). A total of $50 \mu \mathrm{g}$ protein were separated by SDS-PAGE and the membranes probed by western blotting with antibodies for the different cell cycle markers indicated. HepG2 cells were blocked in $\mathrm{G}_{2}-\mathrm{M}_{\mathrm{H}}$ by nocodazole treatment and released in normal or OOS-supplemented medium. Samples were collected at the indicated time points and analyzed as aforementioned by flow cytometry analysis (C) and western blot analysis of key cell cycle markers (D). OOS, Ocoxin ${ }^{\circledR}$ oral solution; AS, asynchronous; DTB, double thyminine block; noc, nocodazole; pHistone, phosphorylated Histone; T, time.

one receiving daily oral treatment with $100 \mu \mathrm{l}$ OOS/20 g body weight and the other, considered the control group, administered $100 \mu \mathrm{l}$ water/20 g body weight. The tumors in the mice receiving OOS treatment grew at a slower rate, as compared with the tumors in the untreated control mice (Fig. 5A). Notably, and due to the aggressive characteristics of the HCC cells in vivo, all mice lost weight throughout the experiment. However, that effect was independent of OOS administration as there were no differences observed in the general behavior of the two groups (Fig. 5B).

To investigate the reason leading to the diminished tumor growth observed in OOS-treated mice, several tumors were resected and fixed subsequent to the sacrifice of the mice, and stained with the proliferation marker Ki-67. When the whole tumor section was observed, a difference in proliferative vs. necrotic regions was identified (Fig. 5C). Thus, when the total necrotic area was measured and normalized to total tumor area, an increase in necrosis was identified in the tumors from OOS-treated mice (Fig. 5D). In addition, OOS induced an evident decrease in the percentage of Ki-67 positive cells present in the proliferative region of the tumor; this was a statistically significant reduction compared with the control untreated tumors ( $\mathrm{P}=0.049$; Fig. 5E).

A second mouse model was used to follow the growth and dissemination properties of HepG2 cells in vivo. For this model, HepG2 cells were transfected with a plasmid coding for the luciferase gene, so that cells carrying the gene would emit light in the presence of luciferin. HepG2 and Huh7 cells positively expressing the luciferase gene, HepG2-Luc and Huh7-Luc, respectively, were isolated and luciferase expression was assessed. One of the clones expressing high levels of the transgene, HepG2-Luc\#22 (Fig. 6A), was selected for additional investigation. This clone, although expressing the luciferase gene, exhibited normal proliferation and cell cycle status compared with the parental cells (data not presented).

HepG2-Luc cells were orthotopically placed in the liver of $\mathrm{BALB} / \mathrm{c}$ nu/nu athymic mice by a small incision in the skin and the direct injection of HepG2 cells into the liver. The correct location of the cells was verified in vivo by bioluminescent imaging (Fig. 6B). The progression of the tumors was followed 

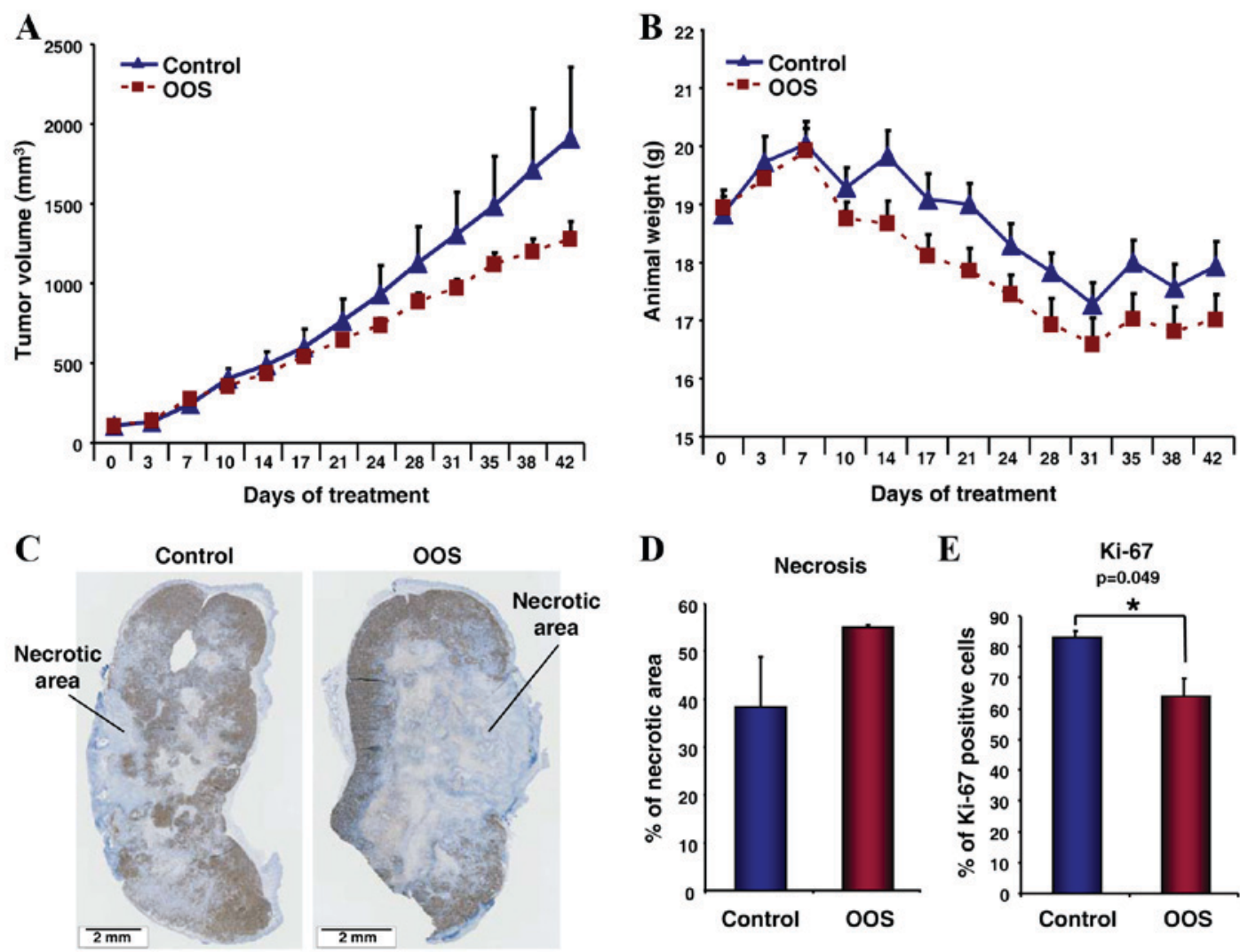

Figure 5. Efficacy of OOS on HCC xenograft models in vivo. (A) OOS interferes with HCC tumor growth. Female BALB/C nu/nu athymic mice were injected with HepG2 cells. Subsequent to three days the tumors were palpable and growing, and were randomly assigned to two groups that were orally treated five days per week with $100 \mu \mathrm{lOOS} / 20 \mathrm{~g}$ body weight, or with vehicle alone. Tumor sizes were measured twice per week. The graph shows the mean tumor volumes \pm standard error of the mean of the mice in each group. (B) Effect of OOS on the weight of the mice, measured twice per week. OOS induced an increase in the necrotic area of the tumor. To establish the proliferative status of the tumors, a Ki-67 marker was used. For each experimental condition $2 / 3$ tumors were randomly selected and processed for immunohistochemical analysis of this marker. Whole tumors were then scanned and the necrotic areas, the non-stained regions, were quantified. (C) Images of representative tumors are presented and (D) the quantification data. (E) The number of Ki-67 positive cells was determined in the proliferative region of the tumors, and the percentage of positive cells was established and represented in the graph. Statistically significant differences are presented $(* \mathrm{P}<0.05)$. HCC, hepatocellular carcinoma; OOS, Ocoxin ${ }^{\circledR}$ oral solution.

by a luciferin injection twice a week. Although the tumors were intrahepatically injected, they rapidly disseminated throughout the abdomen, as demonstrated by imaging (Fig. 6B) and symptoms including an enlarged abdomen due to the accumulation of ascitic fluid (data not presented). In addition, an abdominal inspection of the mice revealed the infiltration of the peritoneal cavity by tumoral masses (Fig. 6C). In general, the liver was normal (Fig. 6D, top image) and at the time of sacrifice only $1 / 14$ of the mice that were analyzed exhibited a mayor hepatic lesion (Fig. 6D, lower image, black arrow).

A total of five days subsequent to injection the mice were randomly assigned into two groups that received water or OOS (200 $\mu \mathrm{l} / 20 \mathrm{~g}$ body weight) by daily oral gavage. The control and the OOS-treated tumors were aggressive; however, the OOS-treated tumors appeared to be less aggressive, as the control mice required sacrifice prior to the OOS-treated mice (Fig. 6E).

\section{Discussion}

Even subsequent to curative resection of HCC in early-stage disease, $\sim 70 \%$ of patients exhibit recurrence after five years (2), increasing the requirement for the development of novel drugs for use in an adjuvant setting. No effective neoadjuvant or adjuvant treatment options that reduce the risk of recurrence are currently available. Additionally, in previous years adoptive immunotherapy and retinoids have been used with some success in HCC $(28,29)$, although the results have been difficult to reproduce.

The present study was conducted in order to explore the potential antitumoral action of OOS in HCC, following several precedents. Firstly, our previous studies demonstrated the antitumoral value of OOS in highly proliferating cells, including breast cancer and acute myeloid leukemia $(13,20)$. Secondly, numerous reports have demonstrated the effects of epigallocatechin-3-gallate on HCC models inducing cell death (30), suppressing hepatocellular carcinoma growth or dissemination. The present study, using two HCC cell lines, observed that OOS induced a decrease in MTT metabolization, indicative of reduced proliferation in the HCC-derived cell lines tested. In addition, combinatory studies using OOS and standard of care drugs used in the therapy of HCC indicated that OOS potentiated the effect of sorafenib, one of the principal drugs used to treat advanced-stage HCC.

Mechanistically, the decrease in MTT metabolization caused by OOS appeared to be cell death-independent, since no major apoptotic population was detected in those conditions. This was a noteworthy result as one of the product 


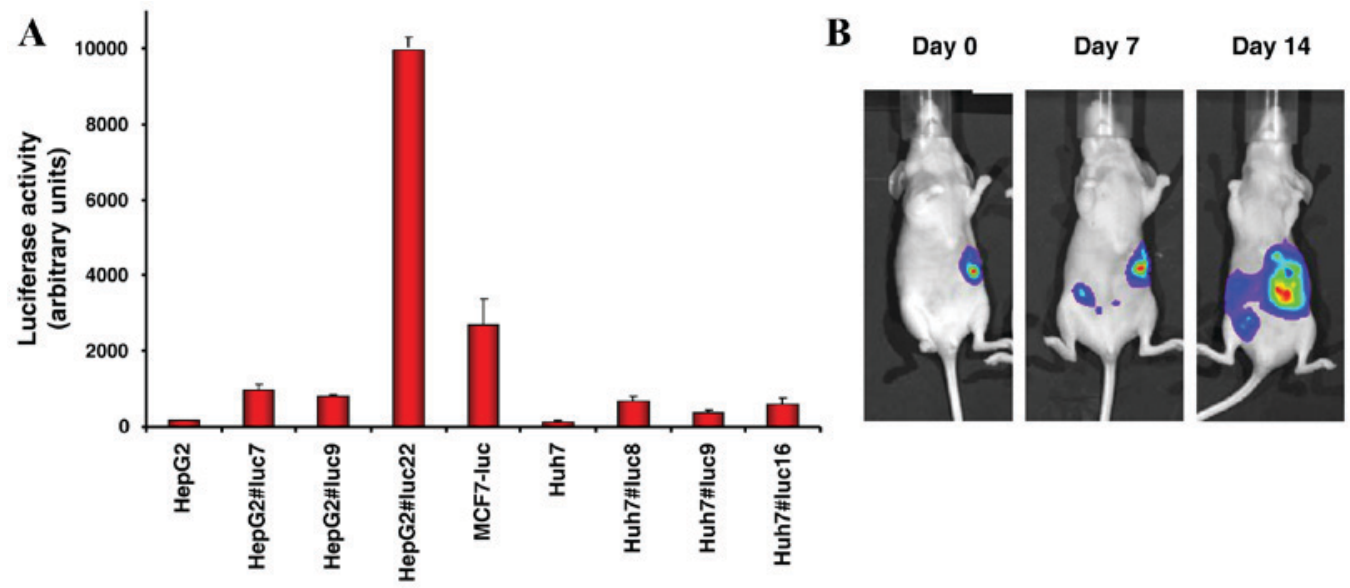

C

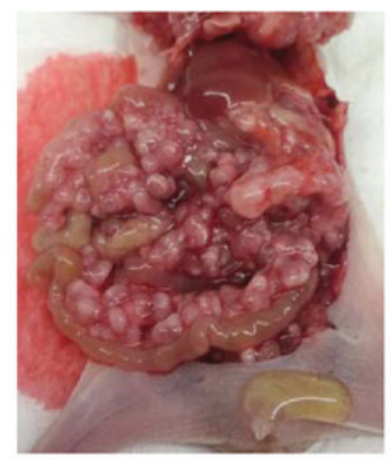

D

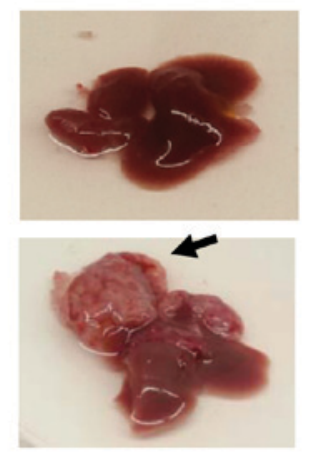

$\mathbf{E}_{1}$

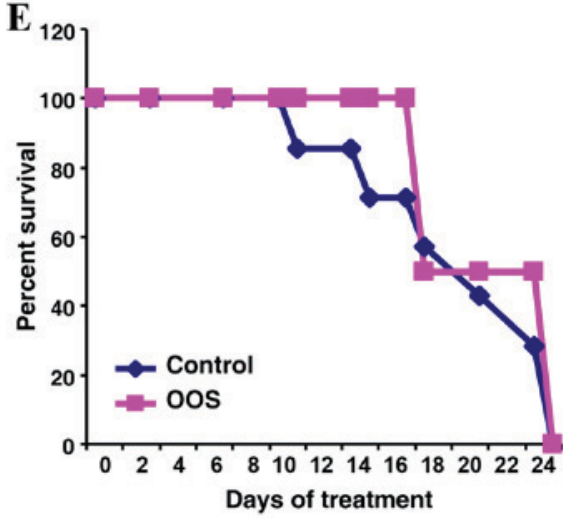

Figure 6. OOS effect on a disseminated HCC tumor model. (A) Generation of HepG2-luc cells. HepG2 and Huh7 cells were transfected with the pCDNA3.1-Luc plasmid and positive clones selected with $450 \mu \mathrm{g} / \mathrm{ml}$ hygromycin, individually harvested and expanded. The expression of the luciferase gene was verified using a Synergy4 multi-mode microplate reader and Gen5 1.05 software. An MCF7-luc clone was analyzed in parallel and used as a positive control. (B) HepG2-Luc cells were implanted directly in the liver of female BALB/C nu/nu athymic mice by surgery. Subsequent to one day, the correct localization of the cells was verified by luciferin detection with the bioluminescence reader Xenogen IVIS 50 using Living Image software. Tumor progression and dissemination were measured twice per week, and representative images of the mice were obtained once per week. (C) Macroscopic image of a representative mouse at the time of sacrifice, exhibiting a large number of small tumoral masses extended throughout the abdominal cavity, and ascitic fluid. (D) Liver status of the mice at the time of sacrifice. The liver of the majority of the mice appeared to be healthy (upper image). Only one of the mice exhibited a liver tumor (black arrow, lower image). (E) Kaplan-Meier curves of the mice. A total of 14 mice were intrahepatically injected with HepG2-Luc cells, and subsequent to five days, randomly assigned into two groups that were orally treated five days per week with $200 \mu 1 \mathrm{OOS} / 20 \mathrm{~g}$ body weight, or with vehicle alone as the control. The graph depicts the time at which the mice required sacrifice due to their general status and abdominal enlargement with ascites. OOS, Ocoxin ${ }^{\circledR}$ oral solution; Luc, luciferase; HCC, hepatocellular carcinoma.

components is epigallocatechin-3-gallate, which has formerly been reported to induce apoptosis in HCC cells (30). The reason for this discrepancy remains unknown, but may be associated with the distinct experimental conditions used in these studies, including the doses of the products employed. While OOS did not induce a noticeable effect on apoptosis, it exhibited a clear effect on cell cycle progression and that is a possible mechanism underlying the antitumoral action of OOS (30). The effect of OOS on cell cycle progression was more evident when analyzing the action of OOS on synchronized cells. In cells synchronized in $\mathrm{G}_{1}$ by a double thymidine block protocol, OOS delayed $\mathrm{G}_{1}-\mathrm{S}$ progression. In addition, the nocodazole block experiments also indicated that OOS delayed the progression from the $M$ to $G_{1}$ phase. The observation that OOS affected progression along various phases of the cell cycle is notable, and suggests that OOS as a companion product may be used with other agents acting on more specific cell cycle phases. Therefore, the action of OOS in HCC appears to involve a delay in cell cycle progression rather than cell cycle arrest, which is in line with the conclusions observed when analyzing other tumor types $(13,20)$.

The data presented in the current study indicates that OOS may be beneficial for patients with HCC, particularly in combination with standard of care drugs. This conclusion is supported by the in vitro data and by the observation that OOS also exerted an antitumoral action when administered to mice carrying xenografted HepG2 cells. The effect of OOS on this in vivo model resulted in a decrease in the rate of tumor growth, suggesting that OOS exerted a cytostatic effect, in line with the in vitro results on the mechanism of action of OOS. In addition, the larger areas of necrotic tumor tissue observed in the tissue samples from OOS-treated mice suggests that this compound may provoke damage to the tumoral tissue, either by acting directly on the tumoral cells or their supporting stroma. These findings are relevant as, together with recent data indicating that patients with terminal HCC exhibited a longer survival when administered OOS (18), it opens the possibility of using OOS to delay tumor progression when used in combination with standard of care therapies. In 
addition, the preventive effect on HCC previously reported for epigallocatechin-3-gallate (8), raises the possibility of using OOS in patients with cirrhosis or patients previously affected with hepatitis B in order to combat the progression of these diseases into HCC. Future studies may assess this hypothesis using models of the aforementioned diseases at pre-tumoral stages.

\section{Acknowledgements}

The plasmid coding for the luciferase gene (pCDNA3.1-Luc) was donated by Dr J. Massagué (Memorial Sloan-Kettering Cancer Center, New York, NY, USA). The present study was supported by Catalysis S.L. and the Ministry of Economy and Competitiveness of Spain (grant no., BFU2012-39151). The present study was supported by a contract from the Spanish Cancer Network (grant no. AECC12/GC02). The Institute of Molecular and Cellular Cancer Biology (CSIC-University of Salamanca) laboratory received support from the European Community through the regional development funding program and from the Fundación Ramón Areces.

\section{References}

1. Forner A, Llovet JM and Bruix J: Hepatocellular carcinoma. Lancet 379: 1245-1255, 2012.

2. Llovet JM, Schwartz M and Mazzaferro V: Resection and liver transplantation for hepatocellular carcinoma. Semin Liver Dis 25: 181-200, 2005.

3. Bruix J, Raoul JL, Sherman M, Mazzaferro V, Bolondi L, Craxi A, Galle PR, Santoro A, Beaugrand M, Sangiovanni A, et al: Efficacy and safety of sorafenib in patients with advanced hepatocellular carcinoma: Subanalyses of a phase III trial. J Hepatol 57: 821-829, 2012.

4. Gish RG, Finn RS and Marrero JA: Extending survival with the use of targeted therapy in the treatment of hepatocellular carcinoma. Gastroenterol Hepatol (N Y) 9 (4 Suppl 2): S1-S24, 2013.

5. Llovet JM, Villanueva A, Lachenmayer A and Finn RS: Advances in targeted therapies for hepatocellular carcinoma in the genomic era. Nat Rev Clin Oncol 12: 408-424, 2015.

6. Mancuso A, Mazzarelli C, Perricone G and Zavaglia C: Sorafenib efficacy for treatment of $\mathrm{HCC}$ recurrence after liver transplantation is an open issue. J Hepatol 60: 681, 2014.

7. Llovet JM, Ricci S, Mazzaferro V, Hilgard P, Gane E, Blanc JF, de Oliveira AC, Santoro A, Raoul JL, Forner A, et al: Sorafenib in advanced hepatocellular carcinoma. N Engl J Med 359: 378-390, 2008.

8. Darweish MM, Abbas A, Ebrahim MA and Al-Gayyar MM: Chemopreventive and hepatoprotective effects of Epigallocatechin-gallate against hepatocellular carcinoma: Role of heparan sulfate proteoglycans pathway. J Pharm Pharmacol 66: $1032-1045,2014$.

9. Lee SI, Kim HJ and Boo YC: Effect of green tea and (-)-epigallocatechin gallate on ethanol-induced toxicity in HepG2 cells. Phytother Res 22: 669-674, 2008.

10. Shimizu M, Shirakami Y, Sakai H, Tatebe H, Nakagawa T, Hara Y, Weinstein IB and Moriwaki H: EGCG inhibits activation of the insulin-like growth factor (IGF)/IGF-1 receptor axis in human hepatocellular carcinoma cells. Cancer Lett 262: 10-18, 2008.

11. Jin J, Chang Y, Wei W, He YF, Hu SS, Wang D and Wu YJ: Prostanoid EP1 receptor as the target of (-)-epigallocatechin-3-gallate in suppressing hepatocellular carcinoma cells in vitro. Acta Pharmacol Sin 33: 701-709, 2012.

12. Zapf MA, Kothari AN, Weber CE, Arffa ML, Wai PY, Driver J, Gupta GN, Kuo PC and Mi Z: Green tea component epigallocatechin-3-gallate decreases expression of osteopontin via a decrease in mRNA half-life in cell lines of metastatic hepatocellular carcinoma. Surgery 158: 1039-1048, 2015.
13. Hernaádez-García S, González V, Sanz E and Pandiella A: Effect of oncoxin oral solution in her2-overexpressing breast cancer. Nutr Cancer 67: 1159-1169, 2015.

14. Hollman PC, Feskens EJ and Katan MB: Tea flavonols in cardiovascular disease and cancer epidemiology. Proc Soc Exp Biol Med 220: 198-202, 1999.

15. Liu L, Hudgins WR, Shack S, Yin MQ and Samid D: Cinnamic acid: A natural product with potential use in cancer intervention. Int J Cancer 62: 345-350, 1995.

16. Yang CS, Chung JY, Yang G, Chhabra SK and Lee MJ: Tea and tea polyphenols in cancer prevention. J Nutr 130 (2S Suppl): S472-S478, 2000.

17. Gomez EV, Perez YM, Sanchez HV, Forment GR, Soler EA, Bertot LC, Garcia AY, del Rosario Abreu Vazquez M and Fabian LG: Antioxidant and immunomodulatory effects of Viusid in patients with chronic hepatitis C. World J Gastroenterol 16: 2638-2647, 2010.

18. Al-Mahtab M, Akbar S, Khan M and Rahman S: Increased survival of patients with end-stage hepatocellular carcinoma due to intake of ONCOXIN ${ }^{\circledR}$, a dietary supplement. Indian J Cancer 52: 443-446, 2015.

19. Dayem Uddin MAI, Mahmood I, Ghosha AK and Khatuns RA: Findings of the 3-Month supportive treatment with ocoxin solution beside the standard modalities of patients with different neoplastic diseases. TAJ 22: 172-175, 2009.

20. Diaz-Rodríguez E, Hernández-García S, Sanz E and Pandiella A: Antitumoral effect of Ocoxin on acute myeloid leukemia. Oncotarget 7: 6231-6242, 2016.

21. Díaz-Rodríguez E and Pandiella A: Multisite phosphorylation of Erk5 in mitosis. J Cell Sci 123: 3146-3156, 2010.

22. Seoane S, Montero JC, Ocaña A and Pandiella A: Breast cancer dissemination promoted by a neuregulin-collagenase 3 signalling node. Oncogene 35: 2756-2765, 2016.

23. de Alava E, Ocaña A, Abad M, Montero JC, Esparís-Ogando A, Rodríguez CA, Otero AP, Hernández T, Cruz JJ and Pandiella A: Neuregulin expression modulates clinical response to trastuzumab in patients with metastatic breast cancer. J Clin Oncol 25: 2656-2663, 2007.

24. Yoshikawa M, Ono N, Yodono H, Ichida T and Nakamura H: Phase II study of hepatic arterial infusion of a fine-powder formulation of cisplatin for advanced hepatocellular carcinoma. Hepatol Res 38: 474-483, 2008.

25. Alberts SR, Reid JM, Morlan BW, Farr GH Jr, Camoriano JK, Johnson DB, Enger JR, Seay TE and Kim GP: Gemcitabine and docetaxel for hepatocellular carcinoma: A phase II north central cancer treatment group clinical trial. Am J Clin Oncol 35: 418-423, 2012.

26. Yata Y, Xue F, Takahara T, Kudo H, Hirano K, Yasumura S, Minemura M, Scanga AE and Sugiyama T: Docetaxel inhibits progression of human hepatoma cell line in vitro and is effective in advanced hepatocellular carcinoma. Hepatol Res 40: 304-310, 2010.

27. Jordan MA, Thrower D and Wilson L: Effects of vinblastine, podophyllotoxin and nocodazole on mitotic spindles. Implications for the role of microtubule dynamics in mitosis. J Cell Sci 102: 401-416, 1992.

28. Muto Y, Moriwaki H, Ninomiya M, Adachi S, Saito A, Takasaki KT, Tanaka T, Tsurumi K, Okuno M, Tomita E, et al: Prevention of second primary tumors by an acyclic retinoid, polyprenoic acid, in patients with hepatocellular carcinoma. Hepatoma Prevention Study Group. N Engl J Med 334: 1561-1567, 1996.

29. Takayama T, Sekine T, Makuuchi M, Yamasaki S, Kosuge T, Yamamoto J, Shimada K, Sakamoto M, Hirohashi S, Ohashi Y and Kakizoe T: Adoptive immunotherapy to lower postsurgical recurrence rates of hepatocellular carcinoma: A randomised trial. Lancet 356: 802-807, 2000.

30. Zhang Y, Duan W, Owusu L, Wu D and Xin Y: Epigallocatechin-3-gallate induces the apoptosis of hepatocellular carcinoma LM6 cells but not non-cancerous liver cells. Int J Mol Med 35: 117-124, 2015. 